\title{
TRB Annual Meeting
}

\section{Evaluating Driver Response to a Dynamic Speed Feedback Sign at a Freeway Exit Ramp Considering the Sign Design Characteristics and Lateral Installation Position --Manuscript Draft--}

Full Title:

Abstract:

Manuscript Classifications:

Manuscript Number:

Article Type:

Order of Authors:
Evaluating Driver Response to a Dynamic Speed Feedback Sign at a Freeway Exit Ramp Considering the Sign Design Characteristics and Lateral Installation Position

Research was conducted at a freeway exit ramp with significant horizontal curvature to evaluate the effectiveness of dynamic speed feedback signs (DSFS) as a speed reduction countermeasure. Several aspects of the DSFS were evaluated, including display size, border type, lateral installation position, and vehicle detection range. Three different full-matrix DSFS were utilized, which included: 15-inch display panel with yellow border, 18-inch display panel with yellow border, and 18-inch display panel with no border. Each sign was individually installed and tested at identical locations near the start of the exit ramp curve, in both the traditional right-side-mount and an alternative forward-mount within the exit gore area. Speed data and message activation location were collected for vehicles approaching and entering into the curve across the various sign test conditions. Overall, the presence of a DSFS positioned near the start of the curve resulted in curve entry speeds that were, on average, 3.5 mph lower than without a DSFS present at the site. The lowest curve entry speeds were observed for cases where the message activated when vehicles were within 250 to $400 \mathrm{ft}$ of the curve. Interestingly, earlier message activation did not contribute to further speed reductions, although later activation substantially diminished the speed reduction effects. Regarding DSFS lateral position, both the side-mounted and forward-mounted DSFS installations resulted in similar curve entry speeds. Furthermore, there were no discernable differences in curve entry speeds between the 15-inch and 18-inch display panels, although the inclusion of a yellow sign border improved performance.

Operations; Traffic Control Devices ACP55

Presentation and Publication

Shakir Mahmud, MS

Timothy Jordan Gates, PhD

Peter T Savolainen, PhD

Babak Safaei, MS

\section{Additional Information:}

Question

\author{
Response
}

The total word count limit is 7500 words

including tables. Each table equals 250

words and must be included in your count. Papers exceeding the word limit may be rejected. My word count is: 
4 by

6 Md Shakir Mahmud, M.Sc.

7 Ph.D. Student

8 Department of Civil and Environmental Engineering

9 Michigan State University

10 East Lansing, MI, 48824

11 Email: $\underline{\text { mahmudmd@msu.edu }}$

Timothy J. Gates, Ph.D., P.E.

14 Associate Professor

15 Department of Civil and Environmental Engineering

Michigan State University

East Lansing, MI 48824

E-mail: gatestim@msu.edu

Peter T. Savolainen, Ph.D., P.E.

MSU Foundation Professor

Department of Civil and Environmental Engineering Michigan State University

East Lansing, MI 48824

E-mail: pete@msu.edu

\section{Babak Safaei}

Graduate Student

Department of Civil and Environmental Engineering Michigan State University

East Lansing, MI, 48824

Email: safaeiba@msu.edu

Words excluding Tables $=6,907$

50 Tables: 2 x 250 = 500; Total Words $=7,407$

51

Date Submitted: July 29, 2021 
Mahmud, Gates, Savolainen, and Safaei

\section{ABSTRACT}

2 Research was conducted at a freeway exit ramp with significant horizontal curvature to evaluate 3 the effectiveness of dynamic speed feedback signs (DSFS) as a speed reduction countermeasure. 4 Several aspects of the DSFS were evaluated, including display size, border type, lateral installation 5 position, and vehicle detection range. Three different full-matrix DSFS were utilized, which 6 included: 15-inch display panel with yellow border, 18-inch display panel with yellow border, and 7 18-inch display panel with no border. Each sign was individually installed and tested at identical 8 locations near the start of the exit ramp curve, in both the traditional right-side-mount and an 9 alternative forward-mount within the exit gore area. Speed data and message activation location 10 were collected for vehicles approaching and entering into the curve across the various sign test 11 conditions. Overall, the presence of a DSFS positioned near the start of the curve resulted in curve 12 entry speeds that were, on average, $3.5 \mathrm{mph}$ lower than without a DSFS present at the site. The 13 lowest curve entry speeds were observed for cases where the message activated when vehicles 14 were within 250 to $400 \mathrm{ft}$ of the curve. Interestingly, earlier message activation did not contribute 15 to further speed reductions, although later activation substantially diminished the speed reduction 16 effects. Regarding DSFS lateral position, both the side-mounted and forward-mounted DSFS 17 installations resulted in similar curve entry speeds. Furthermore, there were no discernable 18 differences in curve entry speeds between the 15-inch and 18-inch display panels, although the 19 inclusion of a yellow sign border improved performance.

21 Keywords: dynamic speed feedback sign, freeway exit ramps, sign size, horizontal curves, lane 22 departure crashes, driver behavior 
Mahmud, Gates, Savolainen, and Safaei

\section{INTRODUCTION}

Freeway interchange ramps serve as the essential connection between a freeway and another freeway or secondary crossroad. Freeway ramps often include a section of horizontal curvature that typically requires a substantial reduction in speed to be safely negotiated. This curvature often leads to an elevated occurrence of vehicles departing the roadway, resulting in a higher rate of crash occurrence than the adjacent mainline freeway segment (1). Annually, approximately 700 fatal crashes occur on freeway ramps in the United States (2). Excessive speed is the most common contributing factor for crashes on freeway ramps (3). Exit ramps are particularly vulnerable to the effects of excessive speeds, due to the deceleration that must occur when transitioning from the mainline freeway to the ramp, which often contain substantial horizontal curvature. Several studies have shown that travel speeds on exit ramps typically greatly exceed the exit ramp advisory speeds (4-7).

Various traffic control strategies have been implemented to reduce speeds and improve the safety performance on freeway ramps, including chevron signs, ramp advisory speed signs, advance curve warning signs, and rumble strips. However, with the exception of rumble strips, such strategies were not found to be effective speed reduction countermeasures on ramps $(4,5)$. A promising countermeasure to reduce curve entry speeds on freeway interchange ramps is the dynamic speed feedback sign (DSFS). DSFS utilize radar to detect the speed of approaching vehicles and display targeted warning messages in real time, which may include the actual speed of the approaching vehicle or other messages such as "SLOW DOWN", "TOO FAST", or "REDUCE SPEED". DSFS have been successfully deployed in work zones, school zones, highspeed arterials, and speed transition zones. However, the use of DSFS at horizontal curves, particularly on freeway exit ramps, remains limited.

\section{LITERATURE SUMMARY}

DSFS have been used to reduce speeds across various road settings, including school zones, residential neighborhoods, high-speed arterials, speed transition zones, work zones, rural highway horizontal curves, and, more recently, freeway interchange ramps. Across these various contexts, the installation of DSFS has resulted in the following ranges of average speed reductions:

- $\quad 1-9 \mathrm{mph}$ in school zones $(8-11)$,

- 1-6 mph in residential neighborhoods $(12,13)$,

- 1-6 mph on high-speed arterials $(8,14-19)$,

- 2-6 mph in speed transition zones $(8,11,20,21)$,

- 1-4 mph on rural highway horizontal curves $(8,11,22,23)$,

- 2-6 mph in rural highway work zones $(11,24,25)$, and

- 2-10 mph in freeway work zones $(11,26-31)$.

Many of these studies also reported substantial improvements in speed limit compliance rates.

In addition to widespread evidence of speed reduction effects across a variety of contexts, DSFS have also demonstrated crash reduction benefits across a limited number of settings. An empirical Bayes analysis on 192 DSFS installed in typical municipal settings on arterial and collector roads showed a significant reduction in crashes of all severity and types, ranging from 32.5 percent to 44.9 percent, with speed-related crashes demonstrating the greatest reductions (32). DSFS have also been well-received by drivers, as evidenced by a survey in California, in which 80 percent of drivers found the information displayed on the DSFS sign to be useful (33).

Evaluation of DSFS at rural highway curves has been relatively limited. A nationwide study by Hallmark et al. (23) investigated the long-term safety and operational effectiveness of 
Mahmud, Gates, Savolainen, and Safaei

DSFS at 22 horizontal curves. The feedback signs were found to produce a significant reduction in average curve entry speeds of approximately $2 \mathrm{mph}$, which was sustained during the 2-year study period. The study also found a 5 to 7 percent reduction in crashes during the first three years after DSFS installation. While the Hallmark et al. study provided evidence that DSFS are effective for reducing speeds and speed-related crashes on rural highway curves, the effects of DSFS on interchange ramp curves were not considered, thereby further supporting the need for additional research.

\section{PROBLEM STATEMENT AND OBJECTIVES}

In light of this research gap, in 2018, researchers at Michigan State University embarked on multiphase research project sponsored by the Michigan Department of Transportation (DOT) to investigate the effectiveness of DSFS at freeway exit ramps possessing significant horizontal curvature. The initial phases of this research project evaluated driver response to various DSFS messaging alternatives and longitudinal installation positions at four freeway interchange ramps. Compared to the existing site condition (without the DSFS), the DSFS reduced curve entry speeds and improved brake response across most test conditions. Overall, considering the combination of both longitudinal sign position and feedback messaging strategy, the greatest speed reduction effects were attained under the following conditions: 1.) the DSFS was positioned at or near the point of curvature (PC) and 2.) the feedback message included the driver's current speed alternating with a "SLOW DOWN" message $(6,7)$. Under these conditions, curve entry speeds were approximately $2 \mathrm{mph}$ lower with the DSFS installed compared to the pre-installation condition. These findings were consistent between the system- and service-interchanges and across all vehicle types (7). However, the sign was found to have little effect on driver behavior when positioned greater than 350-ft upstream of the PC $(6,7)$, perhaps due to drivers disregarding such a premature warning message.

While these evaluations provided encouraging results on the effectiveness of DSFS at freeway exit ramps, it was necessary to expand the evaluation to include additional sign types, sizes and installation configurations to provide comprehensive recommendations on the use of DSFS at freeway exit ramps. To this end, a third and final research phase was undertaken to evaluate the effectiveness of DSFS as a speed reduction countermeasure in this context considering an expanded set of conditions, which included:

- feedback message display size (15-inch vs. 18-inch),

- $\quad$ sign border type (yellow border vs. no border),

- $\quad$ sign placement location (right side-mount vs. forward-mount [i.e., gore area]), and

- vehicle detection range.

\section{FIELD EVALUATION}

To address these objectives, a field evaluation was conducted at a single freeway exit ramp to evaluate the speed reduction effects associated with various DSFS configurations. Several aspects of the DSFS were tested, including size of the display panel, border type, lateral installation location, and location of each subject vehicle when the sign activated (i.e., the sign activation location). Speed data were collected using LIDAR guns for vehicles approaching and entering into the exit ramp curve across the various sign test conditions. A single site was utilized to eliminate site-to-site heterogeneity that would potentially confound the analysis. The following subsections will discuss the various aspects of this evaluation, including test signs, sign installation positions, site description, test conditions, and data collection methods. 
Mahmud, Gates, Savolainen, and Safaei

\section{Test Signs}

Three different speed feedback signs were included in the evaluation, which are displayed in Figure 1. The brightness levels for the message displays were visually matched across the three signs. The general physical characteristics of each sign are noted as follows:

- TraffiCalm sign with an 15-inch full matrix amber feedback display surrounded by a 40inch by 31-inch yellow sheeting border with black "YOUR SPEED" static text,

- TraffiCalm sign with an 18-inch full matrix amber feedback display surrounded by a 48inch by 36-inch yellow sheeting border with black "YOUR SPEED" static text, and

- All Traffic Solution (ATS) sign with an 18-inch full matrix amber feedback display with a white "YOUR SPEED" static panel on top of the display board. This sign did not include an additional border and was consequently a much smaller overall size (30-inch by 20 inch) compared to the TrafficCalm signs.

The radar systems utilized for vehicular detection also varied between the two sign manufacturers, as follows:

- The two TraffiCalm signs utilized a forward-facing radar with a 30-degree cone embedded into the sign face.

- The ATS sign utilized a radar unit that was mounted independently from the sign and employed a more focused 10-degree radar cone, which was intended to better isolate the ramp vehicles and minimize the activation of the sign by the mainline vehicles.

The radar units on all signs were calibrated in the field to achieve vehicle detection ranges that were as similar as possible. However, it was not possible to achieve identical performance due to differences in the radar designs. Specifically, after calibration, the TraffiCalm signs had a typical vehicle detection range (i.e., the location where vehicles would typically be detected by the radar) of approximately 400 feet for passenger cars, which extended up to approximately 600 feet for large trucks. However, the ATS sign, with its more concentrated radar band had a typical vehicle detection range of approximately $600 \mathrm{ft}$ for passenger cars, which extended up to approximately $1,000 \mathrm{ft}$ for trucks.

The differences between the vehicle detection ranges between the two radar systems was a crucial variable for this evaluation, as it relates to the location of the approaching vehicle when the feedback message is initially activated, which may impact driver response. Thus, the sign activation location was estimated for each subject vehicle and was included as a part of the evaluation. Further information on the collection of this data is included in a later section.

During the field evaluations, the signs were powered using a 140 amp-hour portable battery system that was capable of powering the sign for two weeks on a single charge. Each of the signs were able to display a variety of speed feedback messages, and could be programmed to display different messages based on the speed of the approaching vehicle. In accordance with the Michigan DOT draft special provision for speed feedback signs, the three signs were programmed to display the following messages:

- speed number only if the approaching vehicle was below $40 \mathrm{mph}$ and

- speed number of the approaching vehicle alternating every $0.5 \mathrm{sec}$ with a "SLOW DOWN" for vehicles traveling $40 \mathrm{mph}$ or above. 
Mahmud, Gates, Savolainen, and Safaei

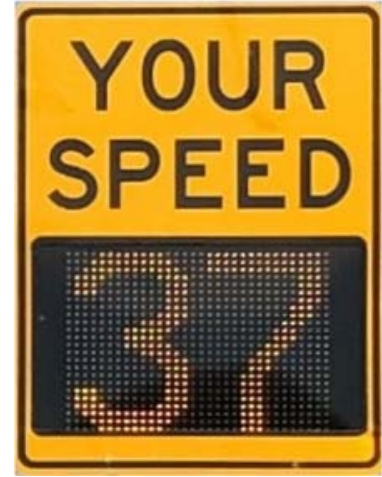

a.) TraffiCalm with 15-inch Display

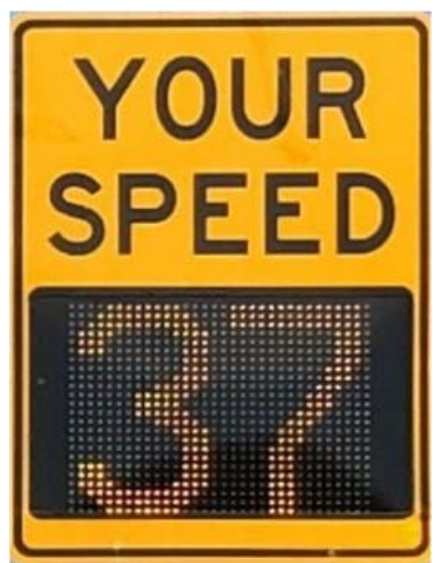

b.)TraffiCalm with 18-in Display c.)ATS with 18-inch Display

FIGURE 1 Dynamic speed feedback signs for field evaluation

\section{Sign Installation Positions}

A primary condition that was evaluated during the earlier phases of this research project was to vary the longitudinal location of the DSFS with respect to the exit ramp curve. As previously noted, during these initial evaluations, the DSFS was consistently found to be most effective when positioned at or near the $\mathrm{PC}$ of the ramp curve, with the sign losing effectiveness at distances of $350 \mathrm{ft}$ or greater upstream from the curve $(6,7)$. However, at many exit ramps locations, the typical right-side-mount DSFS installation near the PC is not feasible due to terrain or obstructions, such as a bridge abutment or guardrail, which would potentially block the radar and/or the motorists' view of the sign.

To that end, a primary variable for the present evaluation was to vary the lateral position of the DSFS with respect to the side of the ramp. Specifically, in addition to the traditional rightside installation at the PC, the DSFS was installed and tested in the ramp gore area, which positioned the sign between the ramp and the mainline freeway and near the green E5-1a Exit sign.

18 In this position, the sign was approximately 40 feet beyond the $\mathrm{PC}$, but directly in front of motorists

19 while approaching the curve in the ramp auxiliary lane. This setup is referred to herein as the 20 "forward-mount" setup, as displayed in Figure 2a along with the traditional right-side-mount setup

21 (herein referred to as "side-mount") example in Figure $2 b$.

22

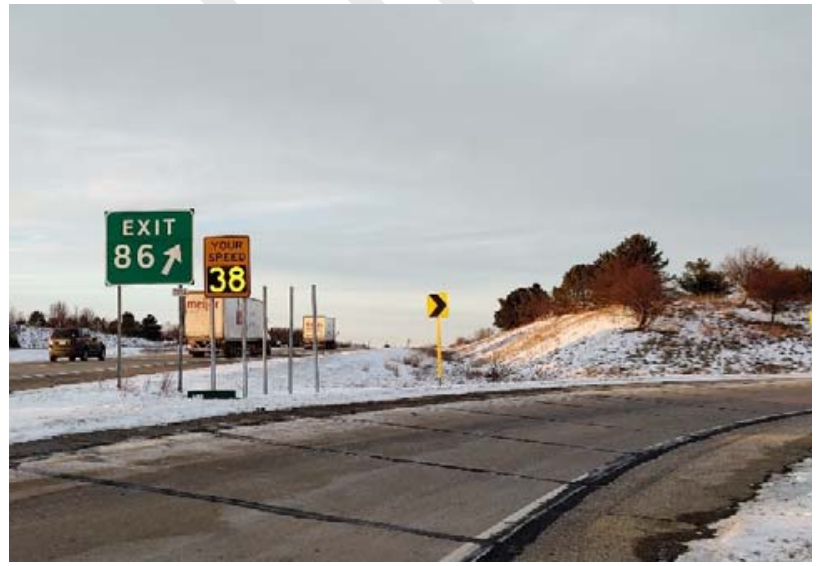

a) Forward-mounted DSFS

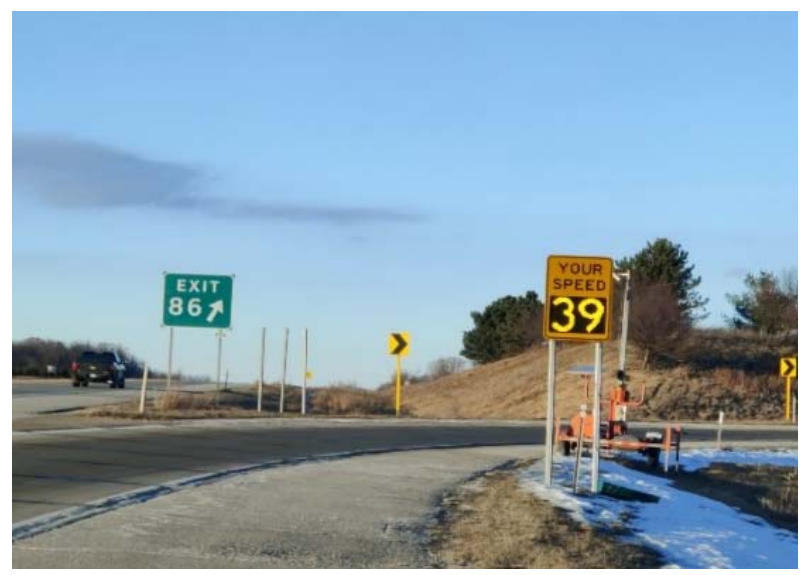

b) Side-mounted DSFS 
Mahmud, Gates, Savolainen, and Safaei

1 Site Description

2 A single freeway exit ramp was selected for the field evaluation based on the following criteria:

- Presence of a horizontal curve with advisory speed of $30 \mathrm{mph}$ or below;

- High frequency of vehicle lane departures at the curve;

- Adequate traffic volumes, while remaining generally uncongested;

- DSFS sign installation capability (i.e., no roadside obstructions or terrain issues); and

- Suitable for data collection.

The selected location was northbound (NB) US-127 to Round Lake Road north of Lansing, 10 Michigan, which is a service interchange with a mainline speed limit of $75 \mathrm{mph}$ and a loop ramp 11 with curve advisory speed of $30 \mathrm{mph}$. This site possessed warning signage that was compliant with the Manual on Uniform Traffic Control Devices (MUTCD), including W13-6, E5-1a, and 13 W1-8R signs. A plan view of the existing signage layout and DSFS locations for this site is 14 provided in Figure 3.

15

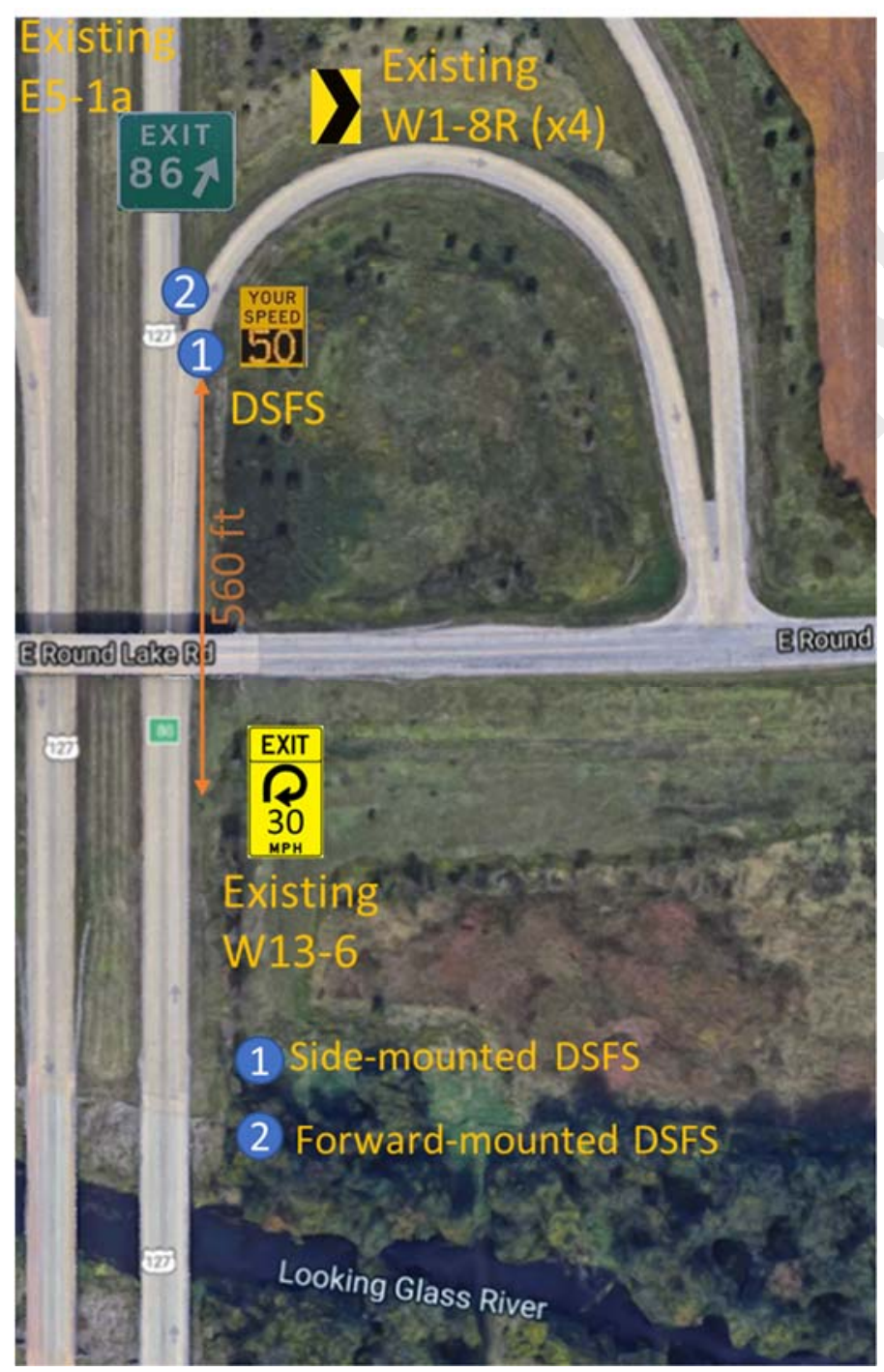


Mahmud, Gates, Savolainen, and Safaei

Sign Test Sequence

2 A total of six DSFS conditions were evaluated and compared to the existing site condition

3 without the DSFS. The sign test conditions and corresponding data collection periods were 4 sequenced as follows:

1. Existing site condition (prior to DSFS installation)

2. Side-mounted 18 -in TrafficCalm sign

3. Side-mounted 15-in TrafficCalm sign

4. Side-mounted 18-in ATS Sign

5. Forward-mounted 18-in TrafficCalm sign

6. Forward-mounted 15-in TrafficCalm sign

7. Forward-mounted 18-in ATS Sign

8. Existing site condition ( 2 months after DSFS removal)

After collection of data under the existing site condition, the DSFS were then installed and evaluated according to the sequence shown above. The DSFS were mounted by MDOT maintenance crews on dual aluminum sign posts, with a 7-ft bottom mounting height from the pavement surface. The existing signage at the site was not modified in any way. The initial DSFS installation remained operational for seven days prior to initiating data collection to allow for dissipation of any driver novelty effects associated with the new traffic control device. For each subsequent change to the DSFS condition, a period of two days was allowed to pass prior to data collection. After completion of all DSFS test conditions, the sign was removed and data were again collected 2-months later under the pre-existing site conditions. All data were collected under dry daylight conditions on weekdays (excluding holidays) during December 2020 and February 2021 of 10:00 AM and 4:00 PM.

\section{Data Collection}

\section{Vehicle Speeds}

Vehicle speeds were continuously tracked for the entire exit ramp lane, beginning from the start of the taper, and continuing to the curve entry point. A sequence of two handheld LIDAR guns (i.e., police laser) operated by technicians from within separate vehicles parked just beyond the shoulder was used. The LIDAR guns were ProLaser III manufactured by Kustom Signals Inc., which can detect vehicular speed and distance three times per second with an accuracy of $\pm 1 \mathrm{mph}$ at a range of 6,000 ft. From a practical sense, each LIDAR gun is typically only utilized over a range of 1,200 feet due to geometry and encroachment of other vehicles.

The upstream and downstream LIDAR technicians were positioned $1,350 \mathrm{ft}$ and $500 \mathrm{ft}$ upstream of the PC, respectively. These locations were selected to be away from any critical points (e.g., start of taper, feedback sign, start of the curve, etc.) to minimize the influence of the data collection vehicle on drivers. Data were collected from the same location for each test condition. The upstream data collector would track each vehicle at least $100 \mathrm{ft}$ beyond the downstream LIDAR technician, at which point the tracking responsibilities were transferred to the downstream technician, who would track each subject vehicle over the remaining distance to the PC. The data collectors communicated via cellular communications to ensure a seamless "hand-off" of the LIDAR speed tracking as each subject vehicle proceeded along the ramp. In doing so, the upstream technician would convey the type and color of each subject vehicle to the downstream LIDAR collector. In order to isolate driver response to the speed feedback sign, only freely flowing vehicles were included. 
Mahmud, Gates, Savolainen, and Safaei

Each LIDAR gun was connected to a laptop using a data transfer cable, which allowed for all measurements to be recorded in real-time using proprietary software. The computer LIDAR recordings included timestamp, distance, and speed for each measurement. After completion of the LIDAR tracking for each subject vehicle, both data collectors added remarks on the type and color of the vehicle, in addition to any other comments, which were later used to combine the two data sets into a continuous speed profile for each subject vehicle. Collecting data using this LIDAR tracking method provides a significant advantage over cameras or pneumatic tubes, as it provides continuous speed measurements over the entire segment of interest, as opposed to spot-speeds at fixed points.

After completion of the LIDAR tracking data collection from the field, both files from the upstream and downstream LIDAR collector were joined using vehicle sequence, type, and color. As the relative distances between the LIDAR collectors and the PC were known, all distances were converted to be relative to the PC. An example representation of the output of this process is shown in Figure 4a. Because LIDAR speeds can't be measured at the same locations on the roadway for every vehicle, it was necessary to convert this data to a series of spot speeds using an interpolation technique in order to allow for speeds to be assessed at specific reference points. The combined raw data were linearly interpolated for every foot using the adjacent speeds. Note that, vehicles with missing large distances in the profile were excluded from this process. Interpolated speeds were then selected at every $50 \mathrm{ft}$ interval starting from the PC, as shown in Figure 4b. Compiling the LIDAR data in this manner provides a robust array of spot speeds at numerous points along the ramp. The data were compiled separately for passenger cars and heavy vehicles (e.g. trucks and buses).

23

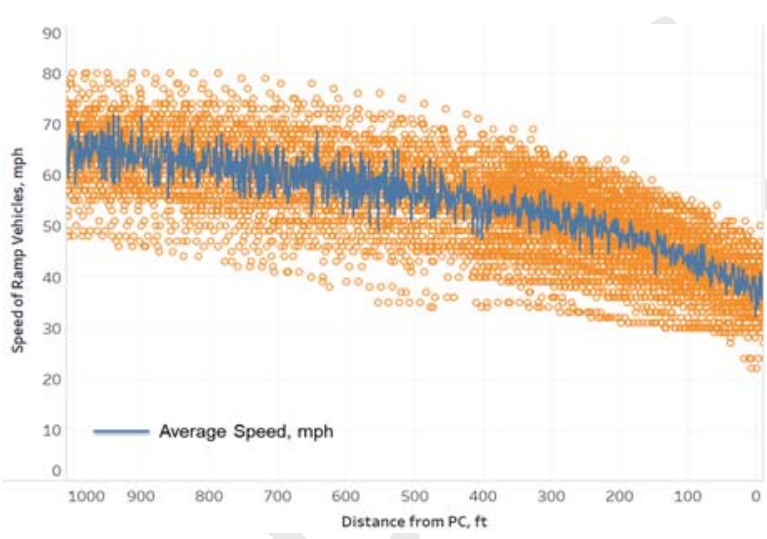

a) Raw LIDAR data ( $\mathrm{n}=203$ vehicles)

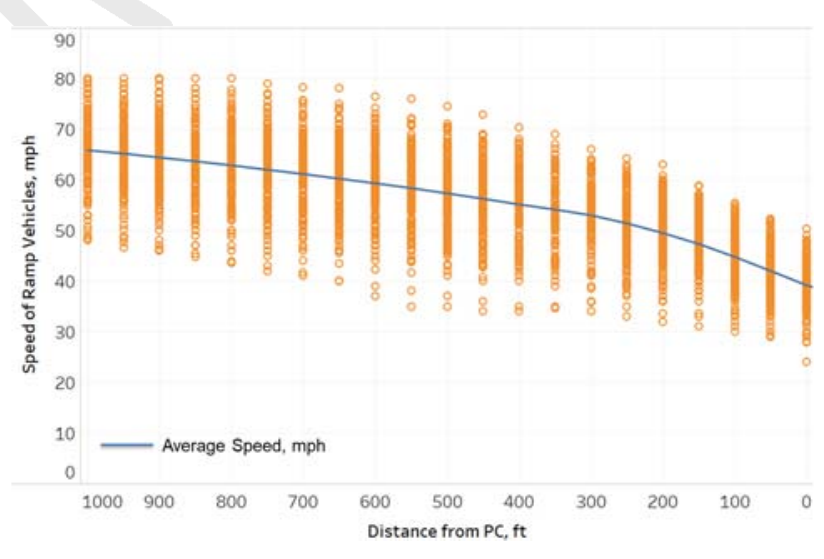

b) LIDAR data interpolated at 50-ft increments

\section{FIGURE 4 Raw and interpolated vehicle speed data}

\section{Feedback Message Activation Location}

It is crucially important for the DSFS to be activated when vehicles are at an appropriate distance in advance of the curve to allow enough time and space to react and decelerate accordingly. As noted previously, the vehicle detection ranges varied between the two radar systems, which subsequently affected when the feedback message would activate for approaching vehicles. To assess the relationship between the message activation point and driver response, the location of each subject vehicle during the initial display of the feedback message was recorded utilizing an elevated video camera temporarily installed on the roadside during each data collection period. 
Mahmud, Gates, Savolainen, and Safaei

1 The videos were later reviewed to extract the location of the vehicle to the nearest 10 feet using a 2 series of reference markers painted on the shoulder. For consistency purposes, all sign activation 3 measurements were referenced to the point of curvature. The sign activation location data were 4 then merged with the LIDAR speed data for each corresponding subject vehicle, and were included 5 as a predictor variable in the subsequent analysis. However, prior to analyzing the data, the sign 6 activation data were first categorized, as follows:

7 - Less than $250 \mathrm{ft}$ upstream of the PC (late activation);

$8 \quad$ - 250 to $400 \mathrm{ft}$ upstream of the PC (normal activation); and

9 - Greater than $400 \mathrm{ft}$ upstream of the PC (early activation).

11 The $250 \mathrm{ft}$ threshold was selected to represent the approximate braking distance necessary for a 12 vehicle to comfortably decelerate (at $11.2 \mathrm{ft} / \mathrm{sec}^{2}$ ) from $60 \mathrm{mph}$ (the approximate $85^{\text {th }}$ percentile 13 speed at this point) to the curve advisory speed of $30 \mathrm{mph}$. The $400 \mathrm{ft}$ threshold was selected as it 14 represented the typical passenger vehicle detection range of the TrafficCalm radar.

\section{Data Summary}

The speed profile data collected for each test condition were joined, organized, and coded into a single file for a comprehensive statistical analysis. The final data set included complete speed profiles for 2,047 vehicle observations, including 1,983 passenger vehicles and 64 heavy vehicles, as shown in Table 1. To check for any obvious trends in the data, sources for potential bias, and data distributions, graphical representations of the data were reviewed and descriptive statistics were compared across each data collection condition. 
Mahmud, Gates, Savolainen, and Safaei

1 TABLE 1 Descriptive Statistics for Ramp Vehicles $(\mathbf{N}=\mathbf{2 , 0 4 7})$

\begin{tabular}{|c|c|c|c|c|}
\hline Parameter & Minimum & Maximum & Mean & Std. Dev. \\
\hline Speed 1,000-ft Upstream of PC, mph & 37.606 & 86.000 & 66.513 & 5.916 \\
\hline Speed 400-ft Upstream of PC, mph & 28.000 & 76.825 & 56.230 & 6.300 \\
\hline Speed 250-ft Upstream of PC, mph & 27.000 & 71.434 & 52.460 & 5.900 \\
\hline Speed At PC, mph & 22.000 & 58.720 & 41.192 & 5.011 \\
\hline Passenger Car & 0 & 1 & 0.969 & 0.174 \\
\hline Heavy Vehicle & 0 & 1 & 0.031 & 0.174 \\
\hline Without DSFS (prior to sign installation) & 0 & 1 & 0.161 & 0.368 \\
\hline \multicolumn{5}{|l|}{ Side-mount DSFS (Sign Activation Location) } \\
\hline 15-in TrafficCalm Sign $(<250 \mathrm{ft})$ & 0 & 1 & 0.062 & 0.241 \\
\hline 15-in TrafficCalm Sign (250-400 ft) & 0 & 1 & 0.022 & 0.148 \\
\hline 15-in TrafficCalm Sign $(>400 \mathrm{ft})$ & 0 & 1 & 0.008 & 0.091 \\
\hline 18-in TrafficCalm Sign $(<250 \mathrm{ft})$ & 0 & 1 & 0.027 & 0.163 \\
\hline 18-in TrafficCalm Sign (250-400 ft) & 0 & 1 & 0.026 & 0.159 \\
\hline 18-in TrafficCalm Sign $(>400 \mathrm{ft})$ & 0 & 1 & 0.021 & 0.143 \\
\hline 18-in ATS Sign $(<250 \mathrm{ft})$ & 0 & 1 & 0.002 & 0.044 \\
\hline 18-in ATS Sign (250-400 ft) & 0 & 1 & 0.018 & 0.131 \\
\hline 18-in ATS Sign $(>400 \mathrm{ft})$ & 0 & 1 & 0.137 & 0.344 \\
\hline \multicolumn{5}{|c|}{ Forward-mount DSFS (Sign Activation Location) } \\
\hline 15-in TrafficCalm Sign $(<250 \mathrm{ft})$ & 0 & 1 & 0.042 & 0.200 \\
\hline 15-in TrafficCalm Sign (250-400 ft) & 0 & 1 & 0.025 & 0.157 \\
\hline 15-in TrafficCalm Sign $(>400 \mathrm{ft})$ & 0 & 1 & 0.031 & 0.174 \\
\hline 18-in TrafficCalm Sign $(<250 \mathrm{ft})$ & 0 & 1 & 0.039 & 0.193 \\
\hline 18-in TrafficCalm Sign $(250-400 \mathrm{ft})$ & 0 & 1 & 0.038 & 0.190 \\
\hline 18-in TrafficCalm Sign $(>400 \mathrm{ft})$ & 0 & 1 & 0.023 & 0.150 \\
\hline 18-in ATS Sign $(<250 \mathrm{ft})$ & 0 & 1 & 0.067 & 0.251 \\
\hline 18-in ATS Sign $(250-400 \mathrm{ft})$ & 0 & 1 & 0.016 & 0.124 \\
\hline 18-in ATS Sign $(>400 \mathrm{ft})$ & 0 & 1 & 0.147 & 0.353 \\
\hline Without DSFS (2 months after removal) & 0 & 1 & 0.088 & 0.283 \\
\hline
\end{tabular}

2

\section{Data Analysis}

Several measures of effectiveness related to vehicle speed were analyzed to determine the effects of the DSFS as a function of sign size/type, lateral installation location, and activation location. The dependent variables for these analyses were selected to assess driver response to the feedback message and included:

- Speed at the point of curvature (i.e., curve entry),

- Speed 250-ft upstream of the point of curvature, and

- Speed 400-ft upstream of the point of curvature.

Additionally, the number of heavy vehicles in the sample was small, which required combining across several test conditions, such that only the DSFS lateral installation position was assessed. 
Mahmud, Gates, Savolainen, and Safaei

All analyses were performed using RStudio. The speed data were analyzed using multiple linear regression, with the form shown in equation 1:

$$
Y_{i}=\beta_{0}+\beta_{1} X_{i 1}+\beta_{2} X_{i 2}+\cdots+\beta_{k} X_{i k}+\varepsilon_{i}
$$

where $Y_{i}$ is the speed at the particular location for vehicle $i, X_{i 1}$ to $X_{i k}$ are independent variables affecting the dependent variables (including DSFS test condition), $\beta_{0}$ is an intercept, $\beta_{1}$ to $\beta_{k}$ are estimated regression coefficients for each independent variable, and $\varepsilon_{i}$ is a normally distributed error term with variance $\sigma^{2}$.

\section{RESULTS AND DISCUSSION}

The multiple linear regression results for speeds approaching and entering the exit ramp curve are show in Table 2. To assist with visualization of the results, Figure 5 displays the parameter estimates and 95 percent confidence intervals for "Speed at PC" across all DSFS test conditions.

Several interesting findings were observed. First, as expected, the speed of vehicles measured 1,000 ft prior to the curve was strongly correlated with speeds approaching and entering the curve, and this effect was stronger at greater distances upstream of the PC. Specifically, this suggests that faster drivers tended to maintain such behaviors regardless of the DSFS presence at the site and is aligned with prior driver behavioral research.

It is also clear that the DSFS tends to have a stronger effect on drivers as they proceed towards the curve. Considering passenger vehicles, speeds measured $400-\mathrm{ft}$ prior to the curve PC were typically only marginally lower with the DSFS in place. By $250-\mathrm{ft}$ prior to the PC, the speeds had become consistently lower with the DSFS in place, particularly for the forward-mount setup. The DSFS showed the greatest effect on speeds measured at the PC (e.g., curve entry point), where speeds were, on average, $3.5 \mathrm{mph}$ lower with a DSFS present. Although the sample of heavy vehicles was small, the DSFS were similarly effective for reducing speeds of heavy vehicles at the curve entry point. It is also worth noting that vehicle speeds collected 2-months after removal of the DSFS had returned to their pre-existing levels. Further discussion of the primary variables of interest, including the effects of lateral sign position, sign size and type, and sign activation location are provided in the sections that follow. Please note that hereinafter, the discussion will primary focus on the results pertaining to speeds measured at the PC, due to the magnitude of the speed reduction effects at this point. 
Mahmud, Gates, Savolainen, and Safaei

1 TABLE 2 Multiple Linear Regression Results for Speeds Approaching and Entering the

2 Ramp Curve

\begin{tabular}{|c|c|c|c|c|c|c|c|}
\hline \multirow{2}{*}{ Parameters } & \multirow[b]{2}{*}{$\begin{array}{l}\text { Activation } \\
\text { Location (ft) }\end{array}$} & \multicolumn{2}{|c|}{ Speed At PC } & \multicolumn{2}{|c|}{$\begin{array}{c}\text { Speed 250-ft } \\
\text { Upstream of PC }\end{array}$} & \multicolumn{2}{|c|}{$\begin{array}{c}\text { Speed 400-ft } \\
\text { Upstream of PC }\end{array}$} \\
\hline & & Estimate & p-value & Estimate & p-value & Estimate & p-value \\
\hline \multicolumn{8}{|c|}{ Vehicle Type: Passenger Cars $(n=1,983)$} \\
\hline Intercept & & 19.461 & $<0.001$ & 8.116 & $<0.001$ & 0.936 & 0.342 \\
\hline Upstream Speed & & 0.367 & $<0.001$ & 0.685 & $<0.001$ & 0.841 & $<0.001$ \\
\hline $\begin{array}{l}\text { Without DSFS } \\
\text { (prior to sign installati }\end{array}$ & & \multicolumn{6}{|c|}{ Base Condition } \\
\hline \multicolumn{8}{|l|}{ Side-mount DSFS } \\
\hline \multirow[t]{3}{*}{ 15-in TrafficCalm } & $<250$ & -3.290 & $<0.001$ & -0.876 & 0.032 & 0.401 & 0.278 \\
\hline & $250-400$ & -4.698 & $<0.001$ & -2.431 & $<0.001$ & -1.100 & 0.053 \\
\hline & $>400$ & -3.594 & $<0.001$ & -2.443 & 0.018 & -1.430 & 0.127 \\
\hline \multirow[t]{3}{*}{ 18-in TrafficCalm } & $<250$ & -3.040 & $<0.001$ & -0.857 & 0.132 & 0.513 & 0.319 \\
\hline & $250-400$ & -4.299 & $<0.001$ & -1.600 & 0.006 & -0.054 & 0.918 \\
\hline & $>400$ & -3.201 & $<0.001$ & -1.377 & 0.035 & -0.407 & 0.493 \\
\hline \multirow[t]{3}{*}{ 18-in ATS Sign } & $<250$ & -2.676 & 0.174 & -1.703 & 0.385 & -1.481 & 0.405 \\
\hline & $250-400$ & -4.554 & $<0.001$ & -2.065 & 0.003 & -1.050 & 0.099 \\
\hline & $>400$ & -4.173 & $<0.001$ & -2.179 & $<0.001$ & -1.365 & $<0.001$ \\
\hline \multicolumn{8}{|l|}{ Forward-mount DSFS } \\
\hline \multirow[t]{3}{*}{ 15-in TrafficCalm } & $<250$ & -2.738 & $<0.001$ & -1.379 & 0.004 & -0.956 & 0.029 \\
\hline & $250-400$ & -4.663 & $<0.001$ & -2.708 & $<0.001$ & -1.929 & $<0.001$ \\
\hline & $>400$ & -3.648 & $<0.001$ & -2.065 & $<0.001$ & -1.566 & 0.003 \\
\hline \multirow[t]{3}{*}{ 18-in TrafficCalm } & $<250$ & -3.538 & $<0.001$ & -0.602 & 0.219 & -0.108 & 0.807 \\
\hline & $250-400$ & -5.247 & $<0.001$ & -2.495 & $<0.001$ & -1.859 & $<0.001$ \\
\hline & $>400$ & -5.050 & $<0.001$ & -2.941 & $<0.001$ & -1.877 & 0.002 \\
\hline \multirow[t]{3}{*}{ 18-in ATS Sign } & $<250$ & -0.500 & 0.211 & 0.009 & 0.982 & 0.042 & 0.908 \\
\hline & $250-400$ & -2.290 & 0.002 & -0.913 & 0.227 & -0.609 & 0.373 \\
\hline & $>400$ & -3.260 & $<0.001$ & -1.347 & $<0.001$ & -0.858 & 0.003 \\
\hline \multirow{2}{*}{\multicolumn{8}{|c|}{$\begin{array}{l}\text { Without DSFS } \\
(2 \text { months after sign removal) }\end{array}$}} \\
\hline & & & & & & & \\
\hline Intercept & $\bar{D}$ & 8.116 & 0.086 & -4.659 & 0.278 & -8.737 & 0.039 \\
\hline Upstream Speed & & 0.516 & $<0.001$ & 0.842 & $<0.001$ & 0.956 & $<0.001$ \\
\hline \multicolumn{2}{|l|}{ Without DSFS } & \multicolumn{6}{|c|}{ Base Condition } \\
\hline Side-mount Setup & & -4.019 & 0.010 & -0.927 & 0.509 & 0.919 & 0.500 \\
\hline Forward-mount Setup & & -3.519 & 0.016 & -0.400 & 0.760 & 0.338 & 0.790 \\
\hline
\end{tabular}


Mahmud, Gates, Savolainen, and Safaei

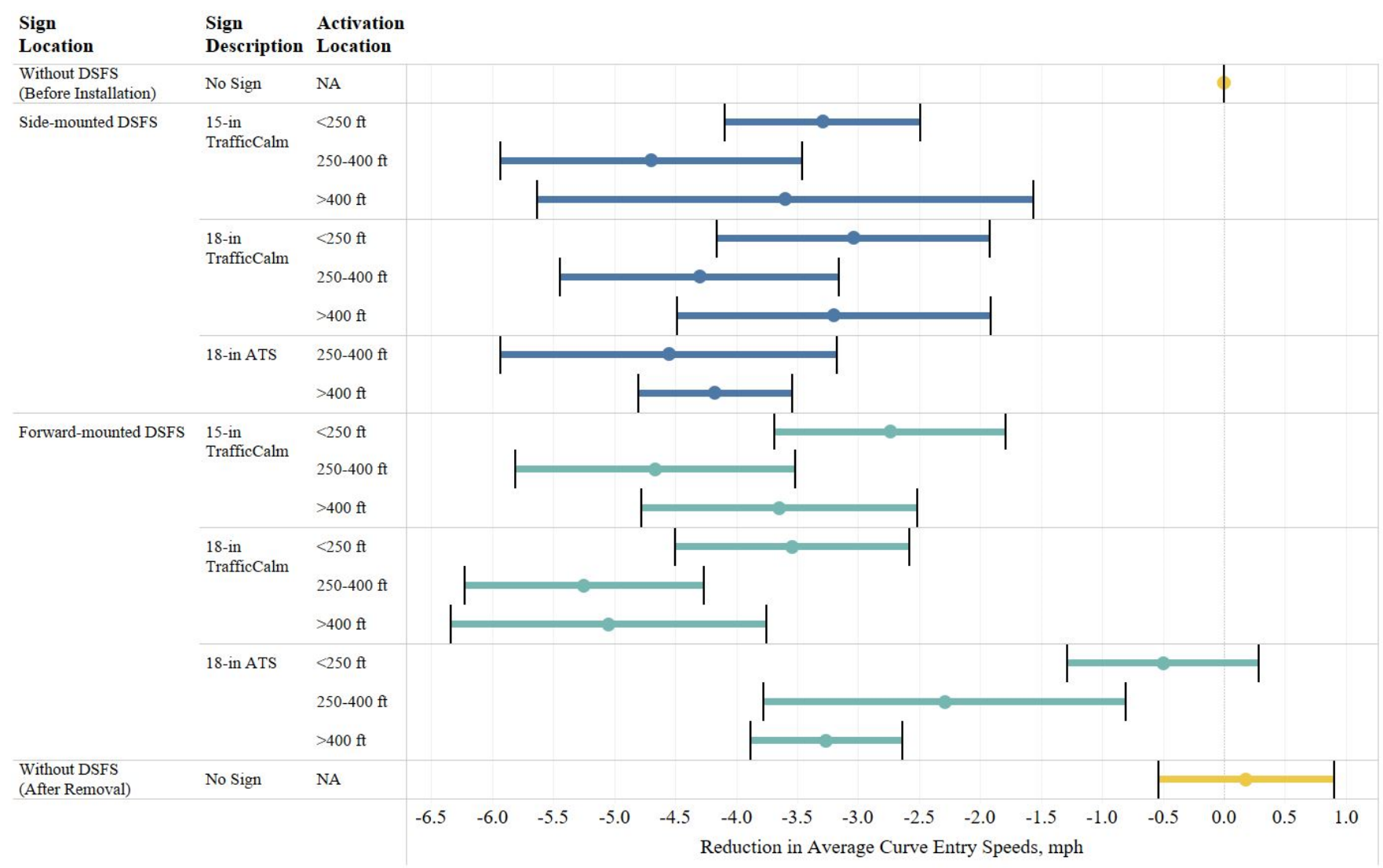

\section{FIGURE 5 Linear regression parameter estimates for reduction in curve entry speed (with 95\% confidence intervals)}

\section{Effect of Sign Lateral Position}

In general, both lateral DSFS installation position elicited a similar effect on curve entry speeds. For passenger vehicles, the reduction in curve entry speed ranged between 3.5 and $4.2 \mathrm{mph}$ for the side-mounted setup, and between 2.4 and $4.5 \mathrm{mph}$ for forward-mounted setup, depending on the sign type and activation location. Although the sample of heavy vehicles was small, speed reductions of 3.5 and $4.0 \mathrm{mph}$ were observed at the curve entry point for the forward-mounted and

11 side-mounted installations, respectively.

\section{Effect of Sign Border and Display Size}

An important aspect of this study was to also compare the sign border type (prominent yellow border vs. no border) and size of the feedback display (15-inch vs. 18-inch). First, considering the size of the feedback display, the 15-inch and 18-inch TraffiCalm signs had a similar effect on curve entry speeds. However, there was evidence of a slight interaction effect between the sign border and lateral position. Specifically, the 18-inch TraffiCalm sign had a slightly stronger speed reduction effect than the ATS sign when utilized in the forward mount position. This may be due to the TrafficCalm's prominent yellow sign border attracting greater attention with the sign posted in the more visually cluttered gore area. Conversely, the ATS sign had a slightly stronger speed reduction effect than the TrafficCalm signs when used in the side-mount condition, which may be due to the more consistent upstream activation of the sign, which is explained in greater detail in the following section. 
Mahmud, Gates, Savolainen, and Safaei

\section{Effect of Message Activation Location}

The message activation location was found to have the strongest relationship with curve entry speed. The greatest reductions in curve entry speeds were observed for cases where the feedback message initially activated when subject vehicles were within 250 to $400 \mathrm{ft}$ of the curve. For such cases, curve entry speed were approximately $4.5 \mathrm{mph}$ lower compared to cases without the DSFS present. Interestingly, the DSFS was found to be slightly, but consistently, less effective at reducing curve entry speeds when initial activation occurred further than $400 \mathrm{ft}$ upstream of the sign.

Across all test conditions, the DSFS was least effective when the feedback message did not initially activate until vehicles were within $250 \mathrm{ft}$ of the curve. This was likely due to drivers not being afforded adequate time to react and respond to the message. In such cases, curve entry speeds were 1.3 to $2.5 \mathrm{mph}$ higher than cases where the sign activated for vehicles that were within 250 to $400 \mathrm{ft}$ of the curve. Generally speaking, the effect of sign activation location was dampened when the sign was side-mounted compared to forward-mounted. Late message activation resulted in particularly poor speed reduction performance for the ATS sign when utilized in the forwardmount position, possibly due to the lack of a conspicuous sign border.

\section{SUMMARY, CONCLUSIONS, AND RECOMMENDATIONS}

The initial phases of this research project evaluated driver response to various DSFS messaging alternatives and installation positions at a series of freeway exit ramps with substantial horizontal curvature. While these prior evaluations provided encouraging results $(6,7)$, it was necessary to evaluate an expanded set of DSFS conditions in order to develop comprehensive recommendations towards their use at freeway exit ramps. To address this objective, a field evaluation was performed which tested several aspects of the DSFS, including physical characteristics (display size and border type), lateral installation location (side-mount vs. forward-mount), and vehicle detection range. A single freeway exit ramp site possessing significant horizontal curvature was utilized to eliminate site-to-site heterogeneity that would potentially confound the analysis.

Three different full-matrix speed feedback signs were utilized during this field evaluation, which included: 1.) TraffiCalm sign with 15-inch display and yellow border, 2.) TraffiCalm sign with 18-inch display and yellow border, and 3.) All Traffic Solutions (ATS) sign with 18-inch display and no border. Each sign was installed and tested in both the traditional right-side-mount and an alternative forward-mount (e.g., gore area) position on the roadside. To assess driver response to the DSFS across the various sign test conditions, handheld LIDAR guns were utilized to track the speeds of vehicles approaching and entering into the exit ramp curve. The location of each subject vehicle when the feedback message was initially displayed was also collected. The salient results of this field evaluation are summarized as follows.

Compared to the existing site condition, installation of a DSFS near the start of the exit ramp curve resulted in lower speeds of vehicles approaching and entering the curve. Generally speaking, the speed reduction effects of the DSFS increased as the vehicles proceeded towards the curve. The greatest effects were observed at the curve PC (e.g., curve entry point), where overall speeds were $3.5 \mathrm{mph}$ lower, on average, with a DSFS present. Reductions in curve entry speeds were observed across all DSFS test conditions, ranging from $0.5 \mathrm{mph}$ to $5.2 \mathrm{mph}$ depending on the test condition. Although the sample of heavy vehicles was relatively small, DSFS were similarly effective for reducing speeds of heavy vehicles at the curve entry point. It is also worth noting that speeds collected 2-months after removal of the DSFS had returned to prior levels, which further supports the effectiveness of the DSFS as a speed reduction countermeasure at exit ramps. 
Mahmud, Gates, Savolainen, and Safaei

Regarding the effect of the lateral installation position, both the side-mounted and forward-mounted DSFS provided a similar level of effectiveness in reducing curve entry speeds, which was consistent for both passenger vehicles and heavy vehicles. Similarly, considering the size of the feedback display, there was no discernable difference in the speed reduction effects between the 15-inch and 18-inch displays. In terms of the sign border, there was evidence of a slight interaction effect between the sign border and lateral position. Specifically, considering the forward-mount position, the prominent yellow sign border produced a stronger speed reduction effect compared to no border, perhaps because due to the greater need for conspicuity with the sign was posted in the more visually cluttered gore area.

The most interesting results were related to the message activation location, which was found to have the strongest effect on curve entry speed across all of the sign-related variables considered. The DSFS were most effective when the feedback message was initially activated for vehicles that were between 250 and $400 \mathrm{ft}$ upstream of the curve, and this finding was consistent across nearly all test conditions. For cases where the feedback message initially activated within this range, curve entry speeds were approximately $4.5 \mathrm{mph}$ lower compared to cases without the DSFS present. Interestingly, the DSFS was found to be slightly less effective at reducing curve entry speeds when initial activation occurred for vehicles further than $400 \mathrm{ft}$ upstream of the curve. This finding is consistent with earlier phases of this research project, which found the DSFS to lose effectiveness when installed $350 \mathrm{ft}$ or further upstream from the curve $(6,7)$, as drivers are more likely to disregard speed warning messages when provided too far in advance of the hazard. Not surprisingly, the DSFS was least effective when the feedback message did not activate until the vehicle was within $250 \mathrm{ft}$ of the curve. This diminished effectiveness was likely due to drivers not being afforded adequate time to react and respond to the message.

The findings from this study further confirm dynamic speed feedback signs to be an effective countermeasure for reducing curve entry speeds at freeway exit ramps, and continued use in this context is recommended according to the following conditions. Specifically, the DSFS should be positioned near the start of the curve, with the radar calibrated such that the message activates when vehicles are at least $250 \mathrm{ft}$ in advance of the curve in order to provide adequate time for drivers to react and decelerate prior to reaching the curve. In terms of sign size, 15 -inch and 18-inch signs were found to be equally effective and may be used interchangeably at freeway exit ramps. A prominent yellow reflective border around the sign is recommended to help improve conspicuity during cases when the sign is activated late and/or when the sign is located in a visually cluttered environment.

When necessary, due to obstructions or terrain issues that would otherwise prohibit the traditional right-side mount, the DSFS may be installed within the gore area of the ramp (e.g., forward-mount setup) between the green Exit sign and the initial chevron depending on ramp configuration and geometry. However, caution should be exercised when positioning the sign in the gore area, due to the increased likelihood that an errant vehicle may collide with the sign in this position. Thus, if possible, installing the sign in the traditional right-side mounting position is preferred.

Finally, while the current and prior phases of this research project have confirmed the effectiveness of DSFS as a speed reduction countermeasure at exit ramps across a variety of contexts, a future evaluation should assess the effectiveness of DSFS towards reducing the frequency/severity of ramp lane departure crashes. 
Mahmud, Gates, Savolainen, and Safaei

DISCLAIMER

2 Funding for this research was provided by the Michigan Department of Transportation (MDOT).

3 This publication is disseminated in the interest of information exchange. MDOT expressly 4 disclaims any liability, of any kind, or for any reason, that might otherwise arise out of any use of this publication or the information or data provided in the publication. MDOT further disclaims any responsibility for typographical errors or accuracy of the information provided or contained within this publication. MDOT makes no warranties or representations whatsoever regarding the quality, content, completeness, suitability, adequacy, sequence, accuracy or timeliness of the information and data provided, or that the contents represent standards, specifications, or regulations.

\section{CONTRIBUTIONS}

The authors confirm contribution to the paper as follows: study concept and design: Timothy Gates, Peter Savolainen, and Md Shakir Mahmud; data collection: Md Shakir Mahmud, Babak Safaei, and Timothy Gates; analysis and interpretation of results: Md Shakir Mahmud and Timothy Gates; draft manuscript preparation: Md Shakir Mahmud and Timothy Gates. All authors reviewed the results and approved the final version of the manuscript.

\section{REFERENCES}

1. McCartt, A. T., V. S. Northrup, and R. A. Retting. Types and Characteristics of RampRelated Motor Vehicle Crashes on Urban Interstate Roadways in Northern Virginia. Journal of Safety Research, Vol. 35, No. 1, 2004, pp. 107-114. https://doi.org/10.1016/j.jsr.2003.09.019.

2. U.S. Department of Transportation. Traffic Safety Performance (Core Outcome) Measures For Michigan. National Highway Traffic Safety Administration (NHTSA). https://cdan.nhtsa.gov/SASStoredProcess/guest.

3. Papadimitriou, E. Meta-Analysis of Crash-Risk Factors in Freeway Entrance and Exit Areas. J. Transp. Eng., 2009, p. 10.

4. Hunter, M., S. Boonsiripant, A. Guin, M. O. Rodgers, and D. Jared. Evaluation of Effectiveness of Converging Chevron Pavement Markings in Reducing Speed on Freeway Ramps. Transportation Research Record: Journal of the Transportation Research Board, Vol. 2149, No. 1, 2010, pp. 50-58. https://doi.org/10.3141/2149-06.

5. Ma, Y., W. Zhang, X. Gu, and J. Zhao. Impacts of Experimental Advisory Exit Speed Sign on Traffic Speeds for Freeway Exit Ramp. PLOS ONE, Vol. 14, No. 11, 2019, p. e0225203. https://doi.org/10.1371/journal.pone.0225203.

6. Gates, T. J., M. S. Mahmud, A. J. Ingle, M. Motz, T. Holpuch, and P. T. Savolainen. Evaluation of Alternative Messages and Sign Locations on Driver Response to a Dynamic Speed Feedback Sign on a Freeway Interchange Ramp. Transportation Research Record, Vol. 2674, No. 12, 2020, pp. 530-541. https://doi.org/10.1177/0361198120959076.

7. Mahmud, M. S., M. Motz, T. Holpuch, J. Hankin, A. J. Ingle, T. J. Gates, and P. T. Savolainen. Driver Response to a Dynamic Speed Feedback Sign on Freeway Exit Ramps Based on Sign Location, Interchange Type, and Time of Day. Transportation Research Record: Journal of the Transportation Research Board, 2021, p. 036119812110152. https://doi.org/10.1177/03611981211015250.

8. Ullman, G. L., and E. R. Rose. Evaluation of Dynamic Speed Display Signs. Transportation research record, Vol. 1918, No. 1, 2005, pp. 92-97. 
Mahmud, Gates, Savolainen, and Safaei

9. O'Brien, S. W., and C. L. Simpson. Use of "Your Speed" Changeable Message Signs in

Transportation Research Record, Vol. 2318, No. 1, 2012, pp. 128-136. https://doi.org/10.3141/2318-15.

10. Ash, K. G. Increasing Speed Limit Compliance in Reduced-Speed School Zones. Brigham Young University - Provo, 2006.

11. Flynn, D. F. B., A. Breck, O. Gillham, R. G. Atkins, and D. L. Fisher. Dynamic Speed Feedback Signs Are Effective in Reducing Driver Speeds: A Meta-Analysis. Transportation Research Record, Vol. 2674, No. 12, 2020, pp. 481-492. https://doi.org/10.1177/0361198120957326.

12. Chang, K. N., M. Nolan, and N. L. Nihan. Measuring Neighborhood Traffic Safety Benefits by Using Real-Time Driver Feedback Technology. Transportation Research Record, Vol. 1922, No. 1, 2005, pp. 44-51. https://doi.org/10.1177/0361198105192200107.

13. Churchill, A. E., and S. Mishra. Speed Feedback Signs as a Tool to Manage Demand for Lower Residential Speeds. Presented at the TAC 2016: Efficient Transportation - Managing the Demand - 2016 Conference and Exhibition of the Transportation Association of Canada, 2016.

14. Ardeshiri, A., and M. Jeihani. A Speed Limit Compliance Model for Dynamic Speed Display Sign. Journal of Safety Research, Vol. 51, 2014, pp. 33-40. https://doi.org/10.1016/j.jsr.2014.08.001.

15. Karimpour, A., R. Kluger, and Y.-J. Wu. Traffic Sensor Data-Based Assessment of Speed Feedback Signs. Journal of Transportation Safety \& Security, 2020, pp. 1-24.

16. Karimpour, A., R. Kluger, C. Liu, and Y.-J. Wu. Effects of Speed Feedback Signs and Law Enforcement on Driver Speed. Transportation Research Part F: Traffic Psychology and Behaviour, Vol. 77, 2021, pp. 55-72. https://doi.org/10.1016/j.trf.2020.11.011.

17. City of Bellevue Transportation Department. Stationary Radar Sign Program 2009 Report.

18. Walter, L., and J. Broughton. Effectiveness of Speed Indicator Devices: An Observational Study in South London. Accident Analysis \& Prevention, Vol. 43, No. 4, 2011, pp. 13551358. https://doi.org/10.1016/j.aap.2011.02.008.

19. Jue, M. J., and J. T. Jarzab. Long-Term Effectiveness of Radar Speed Feedback Signs for Speed Management. Institute of Transportation Engineers. ITE Journal; Washington, Vol. 90, No. 5, 2020, pp. 40-44.

20. Cruzado, I., and E. T. Donnell. Evaluating Effectiveness of Dynamic Speed Display Signs in Transition Zones of Two-Lane, Rural Highways in Pennsylvania. Transportation research record, Vol. 2122, No. 1, 2009, pp. 1-8.

21. Hallmark, S. L., N. Hawkins, and S. Knickerbocker. Use of DSFS as a Speed Transition Zone Countermeasure in Small, Rural Communities. Presented at the 2015 IEEE 18th International Conference on Intelligent Transportation Systems, 2015.

22. Bertini, R. L., C. M. Monsere, C. Nolan, P. G. Bosa, and T. A. El-Seoud. Field Evaluation of the Myrtle Creek Advanced Curve Warning System. Oregon. Dept. of Transportation. Research Unit, 2006.

23. Hallmark, S., N. Hawkins, and O. Smadi. Evaluation of Dynamic Speed Feedback Signs on Curves: A National Demonstration Project. United States. Federal Highway Administration, 2015. 
Mahmud, Gates, Savolainen, and Safaei

24. Mattox III, J. H., W. A. Sarasua, J. H. Ogle, R. T. Eckenrode, and A. Dunning. Development and Evaluation of Speed-Activated Sign to Reduce Speeds in Work Zones. Transportation research record, Vol. 2015, No. 1, 2007, pp. 3-11.

25. Huang, Y., and Y. Bai. Driver Responses to Graphic-Aided Portable Changeable Message Signs in Highway Work Zones. Journal of Transportation Safety \& Security, Vol. 11, No. 6, 2019, pp. 661-682. https://doi.org/10.1080/19439962.2018.1463336.

26. Garber, N. J., and S. T. Patel. Effectiveness of Changeable Message Signs in Controlling Vehicle Speeds in Work Zones. Virginia Transportation Research Council, 1994.

27. Nicholas J. Garber, and Surbhi T. Patel. Control of Vehicle Speeds in Temporary Traffic Control Zones (Work Zones) Using Changeable Message Signs with Radar. TRANSPORTATION RESEARCH RECORD, Vol. 1509, 1995, pp. 73-81.

28. McCOY, P. T., J. A. Bonneson, and J. A. Kollbaum. Speed Reduction Effects of Speed Monitoring Displays with Radar in Work Zones on Interstate Highways. TRANSPORTATION RESEARCH RECORD, 1996, p. 8.

29. Pesti, G., and P. T. McCoy. Long-Term Effectiveness of Speed Monitoring Displays in Work Zones on Rural Interstate Highways. Transportation Research Record: Journal of the Transportation Research Board, Vol. 1754, No. 1, 2001, pp. 21-30. https://doi.org/10.3141/1754-03.

30. Sorrell, M. T., W. A. Sarasua, W. J. Davis, J. H. Ogle, and A. Dunning. Use of Radar Equipped Portable Changeable Message Sign to Reduce Vehicle Speed in South Carolina Work Zones. 2007.

31. Anderson, S. M., J. R. Cunningham IV, J. Liang, and E. J. Fitzsimmons. Evaluation of the Effectiveness of Dynamic Speed Feed Back Signs in Work Zones on High-Speed Kansas Roadways. Presented at the Transportation Research Board 100th Annual MeetingTransportation Research BoardTransportation Research Board, 2021.

32. Wu, M., K. El-Basyouny, and T. J. Kwon. Before-and-After Empirical Bayes Evaluation of Citywide Installation of Driver Feedback Signs. Transportation Research Record: Journal of the Transportation Research Board, Vol. 2674, No. 4, 2020, pp. 419-427. https://doi.org/10.1177/0361198120912243.

33. Tribbett, L., P. McGowen, and J. Mounce. An Evaluation of Dynamic Curve Warning Systems in the Sacramento River Canyon. Western Transportation Institute, Montana State University Bozeman, 2000. 